\title{
„Aber es gibt keine Antisemiten mehr": Eine experimentelle Studie zur Kommunikationslatenz antisemitischer Einstellungen
}

\author{
Heiko Beyer • Ivar Krumpal
}

Zusammenfassung: Die öffentliche Sanktionierung des Antisemitismus nach 1945 hat dazu geführt, dass antisemitische Einstellungen in der bundesdeutschen Öffentlichkeit selten offen geäußert werden, teilweise jedoch im privaten Raum überdauern. Diese Entwicklung wurde von der Antisemitismusforschung zwar theoretisch mit dem Konzept der „Kommunikationslatenz“ aufgearbeitet, methodisch blieben diese Überlegungen jedoch weitgehend folgenlos. Aufgrund des heiklen Charakters antisemitischer Einstellungen ist davon auszugehen, dass sie in Befragungen nicht immer wahrheitsgemäß berichtet und stattdessen häufig sozial erwünschte Antworten gegeben werden. Mittels einer experimentellen Studie zeigen wir, dass die kognitive Aktivierung einer antisemitischen Primärgruppennorm zu einem höheren Maß an selbstberichtetem Antisemitismus führt. Zudem zeigen wir, dass bekannte Determinanten antisemitischer Einstellungen wie Bildung, politische Einstellung und politisches Interesse unter Kontrolle dieses Kommunikationsmechanismus an Erklärungskraft verlieren.

Schlüsselwörter: Antisemitismus · Kommunikationslatenz $\cdot$ Sozial erwünschte Antworten

\section{"But there are no longer any anti-Semites": An experimental study on the communication latency of anti-Semitic attitudes}

\begin{abstract}
Since the public sanctioning of anti-Semitism after 1945 has mostly prevented the articulation of anti-Semitic attitudes in public they outlast in private. This development was taken into account by the theory of anti-Semitism, in particular by the concept of communication latency, but had almost no impact on the data collection process. For the sensitive character of antiSemitic attitudes it is uncertain whether they are reported truthfully in surveys. Respondents are
\end{abstract}

(C) VS Verlag für Sozialwissenschaften 2010

\author{
H. Beyer $(\bowtie)$ \\ Department für Agrarökonomie und Rurale Entwicklung, \\ Georg-August Universität Göttingen, Platz der Göttinger Sieben 5, \\ 37073 Göttingen, Deutschland \\ E-Mail: hbeyer@uni-goettingen.de \\ I. Krumpal $(\bowtie)$ \\ Institut für Soziologie, Universität Leipzig, \\ Beethovenstrasse 15, 04107 Leipzig, Deutschland \\ E-Mail: krumpal@sozio.uni-leipzig.de
}


assumed to answer in a socially desirable manner. Using an experimental setting, we demonstrate that the cognitive activation of an anti-Semitic primary group norm increases the prevalence of self-reported anti-Semitism. In addition, we show that under statistical control of this communication mechanism the explanatory power of established determinants of anti-Semitic attitudes such as education, political attitudes, and political interest decreases.

Keywords: Anti-Semitism $\cdot$ Communication latency $\cdot$ Socially desirable responses

\section{Einleitung}

Es gibt wohl nur wenige Diskurse, die die politische Kultur der Bundesrepublik entscheidender mitbestimmt haben, als jener über den Umgang mit den Verbrechen des Nationalsozialismus. Die Vernichtung der europäischen Jüdinnen und Juden ${ }^{1}$ durch den Vorgängerstaat und der weit verbreitete Antisemitismus in der deutschen Bevölkerung vor 1945 stellten die negative Grundlage eines demokratischen Projektes dar, dessen erste Architekten vor allem darauf bedacht sein mussten, den tradierten Ressentiments entgegen zu arbeiten. „Reeducation“ war hierfür das Schlagwort. Die Antisemitismusforschung hat den daraus folgenden Bruch in der Einstellungsstruktur ${ }^{2}$ des modernen Antisemitismus herausgearbeitet und theoretische Erweiterungen klassischer Erklärungsansätze vorangetrieben. Aktuelle Formen des Antisemitismus, wie „sekundärer Antisemitismus“ (Schönbach 1961; Adorno 1997), „,antisemitische Israelfeindschaft“ oder „Antizionismus“ (Broder 1976; Brumlik 1986) und „struktureller Antisemitismus“ (Haury 2002) werden als Reaktion auf eine öffentliche Sensibilisierung für antisemitische Aussagen und Handlungen verstanden.

Dass die offene Äußerung von antisemitischen Einstellungen und Meinungen nach 1945 mehr und mehr als sozial unerwünscht galt, floss demnach durchaus in die Begriffsund Theoriebildung ein, wurde innerhalb der methodischen Vorgehensweise bislang jedoch nur unzureichend berücksichtigt. Das Äußern von eindeutig antisemitischen Einstellungen wird innerhalb öffentlicher Debatten (vorwiegend informell) sanktioniert. Aus der Methodenforschung ist bekannt, dass die direkte Abfrage von Meinungen und Einstellungen, die gegen soziale Normen verstoßen, zu sozial erwünschtem Antwortverhalten in Befragungen führen kann (Stocké 2004; Schnell et al. 2005; Diekmann 2008; Krumpal 2009, 2010). Befragte machen bei sogenannten ,sensitiven“ oder ,unangenehmen Fragen“ (Van Koolwijk 1969; Lee 1993) zu privaten, strafrechtlich relevanten oder unsozialen Verhaltensweisen oder entsprechenden Einstellungen, häufig falsche Angaben. ${ }^{3}$ Sensitive Fragen werden von den interviewten Personen häufig als indiskret oder

1 Wir verwenden im Folgenden zur sprachlichen Vereinfachung die männliche Form, weisen jedoch darauf hin, dass diese nicht auf die Geschlechtsidentität der bezeichneten Personen schließen lässt.

2 Auch wir beschäftigen uns im vorliegenden Beitrag ausschließlich mit Einstellungen. Unter Einstellungen verstehen wir ,... a psychological tendency that is expressed by evaluating a particular entity with some degree of favor or disfavor" (Eagly und Chaiken 1993, S. 1).

3 Es kann systematisches „Underreporting“ von sozial unerwünschten oder systematisches „Overreporting“"von sozial erwünschten Attributen auftreten. 
gar bedrohlich empfunden, weil sie die Befragten potenziell auffordern, eine Verhaltensweise oder eine Einstellung offenzulegen, die gegen allgemein anerkannte soziale Normen verstößt: „A question is sensitive when it asks for a socially undesirable answer, when it asks in effect, that the respondent admits he or she has violated a social norm" (Tourangeau und Yan 2007, S. 860).

Es stellt sich demnach die Frage, wie Antisemitismus als „heikles Thema“ überhaupt gemessen werden kann, wenn (mindestens) eine Teilmenge der antisemitisch eingestellten Befragten sich der öffentlichen Norm des Anti-Antisemitismus bewusst ist, die Befragung als öffentliche Situation deutet und ihre Meinung demzufolge nicht offen kommuniziert. Die bisherige Forschung kommt in diesem Zusammenhang zu dem Ergebnis, dass antisemitische Einstellungen in Befragungen annähernd wahrheitsgemäß berichtet werden (vgl. Bergmann und Erb 1991a, b). Dieser Schluss wird daraus gezogen, dass ein Teil der Befragten, die das „Thema Juden“ als brisant empfinden, trotzdem durchaus hohe Zustimmungswerte bei den Items zum Antisemitismus aufweisen (Bergmann und Erb 1991b, S. 282). Wir wenden dagegen ein, dass Fragen zur empfundenen Brisanz wohl als gleichermaßen heikel aufgefasst werden können wie jene zum tatsächlichen Antisemitismus. Das „Underreporting“ tritt also möglicherweise schon bei den Fragen zur empfundenen Brisanz des Themas auf, d.h. eine Teilmenge der antisemitisch Eingestellten könnte sowohl bei den eigentlichen Fragen zum Antisemitismus als auch bei denen zur Brisanz sozial erwünschte Antworten geben. Der mögliche Schluss des Forschers von der Abwehrhaltung gegen das „Thema Juden“ auf das Vorliegen antisemitischer Einstellungen, wird auch dem Alltagsbewusstsein nicht schwer fallen.

Anknüpfend an bisherige methodische und sozialpsychologische Studien, die zeigen, dass das Design einer Umfrage und der Fragekontext Auswirkungen auf das Antwortverhalten haben können (Schwarz und Bayer 1989; Strack 1992; Tourangeau und Yan 2007), schlagen wir deshalb ein experimentelles Design vor, mit dem die Effekte sozial erwünschten Antwortverhaltens gezeigt werden können. Die von uns verwendete Methode der Variation der Fragenreihenfolge ist eine Möglichkeit „Kontexteffekte“ (auch „HaloEffekte“; vgl. Nisbett und Wilson 1977) von vorangegangenen Fragen auszunutzen, um die verfügbare Information bei der Beantwortung von nachfolgenden Fragen zu beeinflussen. Die vorliegende experimentelle Studie zeigt einen eindeutigen Kontexteffekt von Fragen, die die Einstellungen der Primärgruppe (hier: die Freunde, die für die Bildung der persönlichen Meinung von Relevanz sind) kognitiv aktivieren auf die Bereitschaft, bei nachfolgenden Fragen eigene antisemitische Einstellungen zu offenbaren. Bevor wir in Abschn. 3 die Ergebnisse jener empirischen Studie berichten, sollen in Abschn. 2 zunächst die theoretische Verknüpfung von Antisemitismustheorie und Befragungspsychologie geleistet und empirisch prüfbare Hypothesen hergeleitet werden.

\section{Theorie und Hypothesen}

\subsection{Begriff und Theorie des Antisemitismus}

Die nach wie vor wohl populärste und umfassendste theoretische Annäherung an den modernen Antisemitismus findet man in den Arbeiten des früheren Frankfurter Instituts 
für Sozialforschung (Adorno et al. 1950; Adorno 1997; Adorno und Horkheimer 1997; Horkheimer 2005) sowie bei Autoren, die sich deren Theorietradition verbunden fühlen (Postone 1988; Claussen 1994; Rensmann 1998). ${ }^{4}$ Hier wird Antisemitismus als Projektion eigener unterdrückter Triebe und als Rationalisierung gesellschaftlicher Verwerfungen der Moderne verstanden und damit entscheidend von anderen Rassismen abgegrenzt. ${ }^{5}$ Mit Thomas Haury (2002, S. 106 ff.) lassen sich daran anknüpfend drei Strukturmerkmale des modernen Antisemitismus festhalten:

a) die Konstruktion des identitären Kollektivs „Juden“, dem als Fremdgruppe negative Attribute zugeschrieben werden, während die Eigengruppe hypostasiert wird; diese Zuschreibungen sind in hohem Maße irreal und emotional aufgeladen (vgl. auch Benz 2004);

b) die Bestimmung ,der Juden“ als Macher der Moderne („Personifizierung“);

c) manichäisches Denken, das die Welt in den Kategorien von Gut und Böse fasst, wobei „die Juden“" das Böse schlechthin verkörpern.

Die Kritische Theorie des Antisemitismus und das damit zusammenhängende Verständnis von Antisemitismus als kohärente Ideologie beziehen sich vorwiegend auf den Vernichtungsantisemitismus des Nationalsozialismus und können schon deshalb nicht ohne weiteres auf aktuelle Phänomene übertragen werden. ${ }^{6}$ Die alten Ressentiments verschwinden aber auch nach 1945 nicht einfach, sondern überdauern bis heute in einem Latenzraum (teils bewusstseins- teils aber auch nur ,kommunikationslatent“; vgl. Bergmann und Erb 1986) und manifestieren sich öffentlich meist nur vermittelt als ,,sekundärer Antisemitismus“, ,,antisemitische Israelkritik“ und ,struktureller Antisemitismus“, die nach 1945 als Reaktion auf die öffentliche Sanktionierung des Antisemitismus entstehen und die angeführten Kriterien nur bedingt erfüllen oder weitere Aspekte umfassen. Diner (2004) spricht in diesem Zusammenhang vom ,,antisemitisierenden Charakter“ einzelner Phänomene.

Bergmann und Erb (1986) reagieren mit ihrem Ansatz der „Kommunikationslatenz“ auf dieses Problem. Der Begriff der „Latenz“ wird dabei explizit für soziale Phänomene verwendet. Kommunikationslatenz unterscheidet sich demnach von Bewusstseinslatenz, indem hier die Funktion für soziale und nicht psychische Systeme untersucht wird (Bergmann und Erb 1986, S.226). Funktional für das politische System sei die Kom-

4 Für einen Theorieüberblick vgl. Fein (1987), Bergmann (1988) und Salzborn (2010).

5 Postone (1988, S. 244) beschreibt den Unterschied von Rassismus und Antisemitismus folgendermaßen: „Nicht nur Ausmaß, sondern auch Qualität der den Juden zugeschriebenen Macht unterscheidet den Antisemitismus von anderen Formen des Rassismus. ... Die Juden stehen für eine ungeheuer machtvolle, unfaßbare internationale Verschwörung.“

6 Es lassen sich jedoch innerhalb der Schriften der Kritischen Theorie durchaus Ansätze finden, die als Ideengeber für die aktuelle Antisemitismustheorie fungieren. So schreiben Adorno und Horkheimer bereits 1947 in der Nachschrift zu den „Elementen des Antisemitismus“: „Aber es gibt keine Antisemiten mehr. Sie waren zuletzt Liberale, die ihre antiliberale Meinung sagen wollten. ... Wurde antisemitische Gesinnung laut, so fühlte sie sich als bürgerlich und aufsässig zugleich“ (Adorno und Horkheimer 1997, S. 226). Antisemitismus tritt vordergründig nicht mehr als reaktionäres Vorurteil auf, sondern als kritische Haltung, die als solche Legitimation besitzen soll. 
munikationslatenz antisemitischer Einstellungen, weil sie es der neuen Bundesrepublik ermöglicht, sich ins westliche Staatenbündnis zu integrieren, ohne sich tatsächlich mit der eigenen Vergangenheit auseinander setzen zu müssen (Bergmann und Erb 1986, S. $227 \mathrm{ff}$.). Medien und Bildungsinstitutionen dienen hierbei als Vermittler der neuen politischen Normen.

Kommunikationslatenz unterscheidet sich von Bewusstseinslatenz darin, dass die antisemitischen Einstellungen, obwohl sie nicht öffentlich geäußert werden, durchaus im Bewusstsein bleiben. Antisemitische Einstellungen drängen demzufolge nach Artikulation jenseits der öffentlichen Ebene (Bergmann und Erb 1986, S.230). Es müssen neue Wege der Äußerung gefunden werden. Die Autoren nennen hierfür zwei Möglichkeiten: „Konsensgruppen“ (z.B. rechtsextreme Vereinigungen) oder „Umwegkommunikation“ (z. B. Antizionismus) (Bergmann und Erb 1986, S. 230).

Legt man den Fokus lediglich auf öffentlich geäußerte klassisch antisemitische Einstellungen oder Meinungen, so übersieht man, dass Antisemitismus kommunikationslatent fortwirken kann. Für Befragungen, die je nach Befragungsumgebung und Wahrnehmung des Befragten sowohl als private als auch als öffentliche Situationen interpretiert werden können, ist nicht auszuschließen, dass ein Teil der antisemitisch eingestellten Befragten ihre antisemitischen Einstellungen nicht berichten, ${ }^{7}$ da diese nur in Konsensgruppen und als Umwegkommunikation geäußert werden. Die erste dieser beiden Varianten ist für unsere Studie von Relevanz.

\subsection{Die Differenz zwischen tatsächlichen und kommunizierten Einstellungen}

Der implizite Verweis von Bergmann und Erb (1986) auf die Bedeutung von Bezugsgruppen (dort: „Konsensgruppen“) für die Kommunikation öffentlich sanktionierter Einstellungen dient uns als Ausgangsbasis für die nähere Bestimmung der wirksamen Mechanismen bei der Meinungsbildung und -kommunikation. Zum Zwecke einer differenzierteren Analyse ist es zunächst sinnvoll, zentrale Termini und Annahmen genauer zu spezifizieren. Mit dem Begriff öffentliche Norm bezeichnen wir die allgemeine und gesamtgesellschaftlich geteilte Norm des Anti-Antisemitismus, deren Verletzung, z. B. durch das öffentliche Äußern antisemitischer Einstellungen, informell und auch rechtlich sanktioniert wird. Dagegen bezeichnet der Begriff Primärgruppennormen empirische Regelmäßigkeiten und Einstellungstendenzen in der Lebenswelt des Befragten (im Sinne von „,behavioral regularities“, vgl. Opp 2001, S. 10714 ff.). Mit Lebenswelt des Befragten ist in erster Linie das unmittelbare soziale Netzwerk von Familie und Freunden gemeint, das man als privat charakterisieren könnte. ${ }^{8}$ Hierbei sind verschiedene Ausprägungen der

7 Stattdessen wäre in Befragungen sozial erwünschtes Antwortverhalten zu erwarten. Damit meinen wir eine systematische Antwortverzerrung in Richtung der öffentlichen Norm des AntiAntisemitismus bei antisemitisch eingestellten Befragten und damit zusammenhängend eine systematische Unterschätzung des tatsächlichen Ausmaßes antisemitischer Einstellungen.

8 In unserer Studie verwenden wir den Begriff der „Primärgruppennorm“ im Sinne einer „Peer Group Norm", um in erster Linie geteilte Einstellungen, Werte und Normen im Freundeskreis des Befragten zu bezeichnen. Die im empirischen Teil untersuchten Einflüsse der „Peer Group“ des Befragten sind als eine Teilmenge des allgemeinen und weiter gefassten Begriffs der „Primärgruppen“ zu betrachten: Der Begriff der „Peer Group“ ist relativ eng gefasst und bezeichnet 
Primärgruppennormen denkbar: 1) Eine Norm des Anti-Antisemitismus, wonach diskriminierende Meinungen über Juden nicht artikuliert werden dürfen. 2) Eine Norm des Antisemitismus, wonach diskriminierende Äußerungen über Juden toleriert oder sogar positiv verstärkt werden. 3) Zwischen diesen beiden Extrempositionen des Einstellungskontinuums können dann verschiedene Einstellungsausprägungen oder -intensitäten in Bezug auf Juden verortet werden. ${ }^{9}$

Auf der Ebene der Meinungsbildung nehmen wir zunächst an, dass sowohl 1) die öffentliche Norm des Anti-Antisemitismus als auch 2) die Primärgruppennorm im Freundeskreis die tatsächliche Einstellung von Akteuren beeinflussen, wobei die Primärgruppennorm einen relativ stärkeren Einfluss ausübt. ${ }^{10}$ Letztere Annahme stützt sich insbesondere auf die kognitionspsychologischen Ansätze von Heider (,Balancetheorie“) und Festinger („Theorie der kognitiven Dissonanz“), die davon ausgehen, dass Einstellungen innerhalb von Kleingruppen (wie zum Beispiel im engeren Freundeskreis) relativ homogen sind. Der Mechanismus liegt hierbei in der Reduktion von Dissonanz begründet. Im Falle von Differenzen in den Einstellungen der Akteure wären psychische Spannungen zu erwarten, die die Akteure als unangenehm empfinden würden (vgl. Heider 1977; Festinger 1978). ${ }^{11}$ Vor diesem Hintergrund kann die Primärgruppennorm im Freundeskreis als ein besserer Prädiktor für die tatsächlichen Einstellungen der Akteure angesehen werden als die

in erster Linie das soziale Netzwerk von Jugendlichen. Dagegen kann der Begriff der „Primärgruppen" neben dem Freundeskreis des Befragten auch andere engere Kontakte, wie etwa die Mitgliedschaft in einer Familie, umfassen. Wir verwenden in der vorliegenden Studie durchgehend den allgemeinen Begriff und verweisen in diesem Zusammenhang auf die Möglichkeit von alternativen Konzeptspezifikationen in zukünftigen Studien (z. B. eine Operationalisierung über Familieneinflüsse).

9 In der vorliegenden Arbeit wird angenommen, dass entsprechende Einstellungen und Normen in vielen Fällen in der „Peer Group“ des Befragten vorliegen und somit sinnvoll mit standardisierten Messinstrumenten empirisch untersucht werden können. Einige neuere, qualitative Studien mittels Gruppendiskussionen (vgl. Scherr und Schäuble 2008) geben Hinweise auf z.T. diffuse und nur wenig kohärente Einstellungen in Bezug auf Juden. Die Autoren betonen in diesem Zusammenhang den Unterschied von (anti-)antisemitischem Selbstverständnis der Befragten einerseits, und kommuniziertem Antisemitismus andererseits. Bei der Verwendung eines standardisierten Messinstrumentes würde man annehmen, dass sich Befragte mit diffusen Einstellungen eher in der Mitte der Antwortskala einordnen würden.

10 Um genauere Aussagen darüber zu treffen, ob bei divergenten Normen die Norm der Außengruppe (hier: die „öffentliche Norm“) oder die Norm der Innengruppe (hier: die „Primärgruppennorm") befolgt wird - welche Gruppe also tatsächlich als Bezugsgruppe gelten kann (vgl. Merton 1957, S. 281 ff.) - hängt unter anderem davon ab, welchen Status das Individuum innerhalb der Innengruppe einnimmt. Vor allem mittlere Statuspositionen geben Anreiz zu normkonformem Verhalten in der Innengruppe (vgl. Homans 1974, S. 319 ff.; Phillips und Zuckerman 2001). In unserer Arbeit nehmen wir die Innengruppen als primäre Bezugsgruppen an, gestehen aber auch der Außengruppe meinungsbildende Wirkung zu. Natürlich können die Normen beider Gruppen auch identisch sein.

11 So würden anti-antisemitisch eingestellte Akteure mit einer hohen Wahrscheinlichkeit aus einer antisemitisch eingestellten Gruppe ausgegrenzt werden oder würden von vornherein aus Gründen kognitiver Dissonanz (Festinger 1978) einen anderen Freundeskreis wählen. 
öffentliche Norm, deren Zusammenhang mit den tatsächlichen Einstellungen der Akteure als vergleichsweise schwach angenommen wird.

Auf der Ebene der Meinungskommunikation nehmen wir dagegen an, dass die relative Wirkung von öffentlicher Norm und Primärgruppennorm auf die kommunizierte Einstellung von Akteuren durch situative Aspekte moderiert wird. Wenn keine Inkongruenz zwischen den Normen der Primärgruppe und der öffentlichen Norm des Anti-Antisemitismus vorliegt, wenn also die Primärgruppe und annahmegemäß mit einer hohen Wahrscheinlichkeit auch die betreffende Person nicht antisemitisch sind, dann wird diese Person sowohl in öffentlichen als auch privaten Situationen entsprechende Einstellungen kommunizieren. Es wird dann zu keiner Differenz zwischen tatsächlicher und kommunizierter Einstellung kommen.

Wenn dagegen eine Inkongruenz zwischen öffentlicher Norm und Primärgruppennorm vorliegt, die Primärgruppe also hinreichend antisemitisch ist, dann ist zwischen öffentlichen und privaten Situationen klar zu differenzieren. In öffentlichen Situationen werden hinreichend antisemitisch eingestellte Akteure weniger antisemitische Einstellungen als tatsächlich vorliegend kommunizieren und stattdessen eher dazu neigen, ihre kommunizierten Einstellungen an der öffentlichen Norm des Anti-Antisemitismus auszurichten. Durch die angenommene Verstärkung des Einflusses der öffentlichen Norm des Anti-Antisemitismus (relativ zum Einfluss der Primärgruppennorm) auf die kommunizierte Einstellung in öffentlichen Situationen ist somit zu erwarten, dass sich die Differenz zwischen der tatsächlichen und der kommunizierten Einstellung ${ }^{12}$ bei antisemitisch eingestellten Akteuren vergrößert. In privaten Situationen wird bei Akteuren mit antisemitischen Einstellungen und entsprechender Primärgruppennorm die öffentliche Norm des Anti-Antisemitismus hingegen keinen oder einen nur schwachen Einfluss auf die kommunizierte Einstellung ausüben und zu keiner Differenz zwischen tatsächlicher und kommunizierter Einstellung führen. So ist zu erwarten, dass antisemitisch eingestellte Akteure in privaten Situationen eher dazu neigen werden, ihre antisemitischen Einstellungen zu kommunizieren.

Wird nun im Rahmen einer empirischen Antisemitismusstudie die Befragungssituation von den Befragten als öffentliche Situation gedeutet, wovon wir zunächst ausgehen, dann ist gemäß der obigen Argumentation eine Differenz zwischen tatsächlicher und kommunizierter Einstellung bei Befragten mit antisemitischen Einstellungen zu erwar-

12 Im Vergleich zu selbstberichtetem Verhalten („factual questions“), ist das Konzept eines „wahren Wertes“ bei Einstellungsfragen (,non-factual questions“) schwerer zu rechtfertigen, da ein objektiver Vergleichsmaßstab nicht vorliegt: „Whether 'true values' exist for subjective states, such as knowledge, opinions, and feelings, is controversial" (Groves et al. 2004, S. 260). Trotzdem gibt es aber auch im Zusammenhang mit Meinungs- und Einstellungsmessungen namhafte Positionen, die dafür plädieren, sozial erwünschte Antworten als systematische Abweichungen von „true scores“ (im Sinne eines ,target construct“, eines stabilen, innerpsychischen Konstrukts) zu denken (Schaeffer und Presser 2003). Somit ist die Differenzierung zwischen der (unbeobachteten) tatsächlichen Einstellung und der (beobachteten) kommunizierten Einstellung in erster Linie analytischer Art und dient insbesondere dazu, die Idee einer ,systematischen Antworttendenz" klarer auf den Punkt zu bringen. 
ten. Diese Differenz wird von uns als das Ausmaß sozial erwünschten Antwortverhaltens interpretiert. $^{13}$

$\mathrm{Da}$ die Antworten in einer empirischen Antisemitismusstudie streng genommen nur den kommunizierten Antisemitismus abbilden, sollte die Befragungssituation in der Art gestaltet werden, dass sie von antisemitisch eingestellten Befragten als private Situation interpretiert wird und somit die Differenz zwischen tatsächlicher und kommunizierter Einstellung minimiert wird. Eine Minimierung dieser Differenz wäre wiederum gleichbedeutend mit einer Verringerung des Ausmaßes sozial erwünschten Antwortverhaltens und einer Erhöhung der Validität der Messung antisemitischer Einstellungen im Rahmen einer Befragung (vgl. Tourangeau et al. 2000, S. 266 f.). Zu diesem Zweck schlagen wir vor, mithilfe von „Kontexteffekten“ vorangegangener Fragen die jeweilige Primärgruppennorm kognitiv zu aktivieren, um private Einflüsse bei der Beantwortung von heiklen Fragen zum Thema Antisemitismus zu verstärken.

\subsection{Antwortprozess und Kontexteffekte}

Befragungspsychologische Studien zu Kontexteffekten bei der Einstellungsmessung ${ }^{14}$ zeigen, dass eine gezielte Variation des Fragekontexts oder der Fragereihenfolge zu Unterschieden in der Beantwortung von Folgefragen führen kann (Bishop et al. 1984; Strack et al. 1991; Schwarz und Sudman 1992). Als „Kontexteffekt“ bezeichnet man allgemein den Einfluss vorangegangener Fragen oder des Antwortprozesses bei vorangegangenen Fragen auf den Antwortprozess nachfolgender Fragen (Strack 1992; Tourangeau et al. 2000; Rauhut et al. 2009). Die vorangegangenen Fragen haben hierbei zwei Funktionen (Strack 1992, S. 33): 1) Sie aktivieren über einen ,priming effect ${ }^{\text {" } 15}$ kognitive Strukturen

13 Mit dem Begriff, ,sozial erwünschtes Antwortverhalten“" meinen wir eine systematische Antworttendenz in Richtung der in der BRD herrschenden öffentlichen Norm des Anti-Antisemitismus bei antisemitisch eingestellten Befragten. Die Richtung der Differenz zwischen tatsächlichen und kommunizierten Einstellungen ist somit im Rahmen von Befragungen vorhersagbar. Im Rückgriff auf eine befragungspsychologische Terminologie erwarten wir ein systematisches „Underreporting“ von antisemitischen Einstellungen (vgl. Krumpal 2009, 2010). Aufgrund der erwarteten Unterschätzung des tatsächlichen Ausmaßes antisemitischer Einstellungen wird im Rahmen eines experimentellen Vergleichs verschiedener Befragungskontexte zudem angenommen, dass die validere Messung zu einem relativ höheren Ausmaß an selbstberichteten Antisemitismus führen wird, d.h. ein relativ höheres Ausmaß an sozial unerwünschten Antworten generiert („,more-is-better assumption“, vgl. hierzu Tourangeau und Yan 2007).

14 Ein bekanntes Modell zur Beantwortung von Einstellungsfragen findet sich bei Tourangeau und Rasinski (1988) und Tourangeau et al. (2000). In Interviews kann der kognitive Prozess der Beantwortung von Einstellungsfragen in vier Stufen unterteilt werden: 1) Informationsaufnahme und Interpretation des Frageinhalts, sowie die automatische Aktivierung einer Einstellungsstruktur im Langzeitgedächtnis, 2) Abrufen relevanter Informationen, 3) Beurteilung der erinnerten Informationen, und 4) Auswahl und Wiedergabe der Antwort.

15 Studien der Kognitionspsychologie zeigen, dass Befragte bei der Bildung einer Antwort nicht alle potenziell relevanten Informationen aus dem Gedächtnis abrufen, sondern die kognitive Anstrengung abbrechen, sobald genügend relevante Inhalte für eine Antwortbildung aktiviert wurden (Schwarz und Bayer 1989, S. 4). Die Antwort basiert somit in erster Linie auf Informationen die zuerst aktiviert werden und für die Beantwortung der Frage relevant sind. Je leichter 
im Gedächtnis des Befragten, sodass die aktivierten Informationen zu einem späteren Zeitpunkt leichter verfügbar sind (Aktivierungsfunktion). Dieser Aktivierungsprozess geschieht häufig automatisch und unbewusst: „This information is either contained in the previous question or activated by the respondent in order to generate an answer to the previous question. Merely by having been activated before, the information subsequently becomes more accessible. Moreover, this process can occur without the respondent's awareness [...]“ (Strack 1992, S. 25 f.). 2) Sie liefern zudem eine Informationsbasis im Lichte derer die nachfolgenden Fragen interpretiert und gedeutet werden (Informationsfunktion). Die Informationen und Erinnerungen, die durch die Beantwortung der vorangegangenen Frage aktiviert wurden, sind zugänglicher und wirken auf die Beantwortung der Folgefragen. Sie dienen als Referenzrahmen, an dem sich nachfolgende Kognitionen und Antworten orientieren (vgl. Tourangeau 1992, S. 38): „By making a particular dimension, norm, or standard of comparison salient, context can also alter how respondents make their judgment. For example, prior items may trigger the application of a norm that is carried over to a later item [...].“

Die Erinnerung relevanter Informationen wird beeinflusst vom Inhalt vorangegangener Items und der Verfügbarkeit früherer Überlegungen (Tourangeau 1992, S. 36). Die gezielte Manipulation der Fragereihenfolge nutzt dieses bekannte Phänomen, um über vorangegangene Items inhaltlich relevante Informationen zur Beantwortung nachfolgender Fragen kognitiv verfügbar zu machen (Schwarz et al. 1989; Schwarz 1991). Solche „Kontexteffekte“ könnten nun im Rahmen von empirischen Studien zu Antisemitismus genutzt werden, um Primärgruppennormen kognitiv zu aktivieren und die Beantwortung von heiklen Fragen zu Einstellungen über Juden in Richtung einer stärkeren Konsistenz mit diesen Primärgruppennormen, deren Zusammenhang mit den tatsächlichen Einstellungen der Befragten als stark angenommen wird, zu beeinflussen.

\subsection{Der Einfluss der Primärgruppennorm auf den kommunizierten Antisemitismus}

Antworten auf Fragen nach den eigenen antisemitischen Einstellungen können im Prinzip auf der Basis unterschiedlicher, potenziell relevanter Informationen und kognitiver Inhalte entstehen (durch Medien transportierte öffentliche Normen; eigene Erfahrungen mit Juden; gesamtgesellschaftliche Tabus; Stereotypen; soziale Normen im Verwandtenund Freundeskreis). Den befragungspsychologischen Annahmen gemäß ist zu erwarten, dass aus der Menge aller potenziell relevanten Informationen diejenige Teilmenge in die Antwortbildung einfließt, die zum Zeitpunkt der Fragebeantwortung besonders leicht verfügbar ist: „Die theoretische Grundlage der Strategie der Informationsaktivierung besteht in dem Befund, dass Personen eine gegebene relevante Information mit umso größe-

relevante Informationen erinnert werden, desto höher ist die Wahrscheinlichkeit, dass sie in die Antwortbildung einfließen. Die Leichtigkeit der Erinnerung einer bestimmten Information ist wiederum eine Funktion der verstrichenen Zeit seit ihrer letzten Aktivierung. Die Leichtigkeit mit der eine vorangegangene Beurteilung oder Wissensstruktur ins Bewusstsein gerufen wird, wird auch als Verfügbarkeit bezeichnet (Feldman 1992, S. 51). Gedächtnisinhalte, über die der Befragte erst vor kurzem nachgedacht hat, sind mit einer höheren Wahrscheinlichkeit kognitiv verfügbar als Inhalte deren letzte Aktivierung aus dem Gedächtnis bereits längere Zeit zurückliegt. Dieses Phänomen wird häufig als ,priming effect“ bezeichnet (vgl. Sloman et al. 1988). 
rer Wahrscheinlichkeit zur Urteilsbildung heranziehen, je leichter sie ihnen in den Sinn kommt" (Schwarz und Bayer 1989, S. 16).

Bei der Messung von antisemitischen Einstellungen könnte der Fragekontext nun derart gestaltet werden, dass die Beantwortung der Items zum eigenen Antisemitismus stärker in den privaten Kontext des Befragten eingebettet ist und stärker im Einklang mit den Primärgruppennormen steht, die diesen privaten Kontext aufspannen. Konkret kann durch eine gezielte Variation der Fragereihenfolge die temporäre kognitive Verfügbarkeit von Primärgruppennormen, welche stark mit den antisemitischen Einstellungen von Ego zusammenhängen, erhöht und somit die Beantwortung der Folgefragen zum eigenen Antisemitismus beeinflusst werden. Den Befragten werden keine festen Normen und Bewertungen vorgegeben (wie im Falle der öffentlichen Norm), vielmehr werden die Primärgruppennormen direkt erfragt. Hierdurch wird ein selbst generierter Referenzanker gebildet, den die Befragten als Grundlage für die Beantwortung der Folgefragen nutzen können, in denen sie aufgefordert werden, ihre antisemitischen Einstellungen zu berichten. So könnten Befragte mit hinreichend antisemitischen Einstellungen eher bereit sein, sozial unerwünschte Antworten zu geben, indem sie antisemitischen Items zustimmen, wenn in vorhergehenden Fragen eine (antisemitische) Primärgruppennorm kognitiv aktiviert wurde, die mit der eigenen Meinung über Juden konsistent ist. ${ }^{16}$ Durch die kognitive Aktivierung würde die Primärgruppennorm für das Antwortverhalten in nachfolgenden Fragen an Relevanz gewinnen und die öffentliche Norm in den Hintergrund drängen und somit deren Wirkung auf das Antwortverhalten abschwächen. ${ }^{17}$

Aus diesen Überlegungen lassen sich zwei empirisch prüfbare Hypothesen ableiten. Diese werden im Rahmen eines experimentellen Designs überprüft, in dem die Experimentalgruppe die Fragen zur Primärgruppennorm vor den Fragen nach den eigenen

16 Die Anpassungsprozesse im Antwortverhalten geschehen nicht aufgrund von Sanktionsbefürchtungen. Die Freunde sind bei der Befragung nicht anwesend und können somit eine etwaige Normverletzung des Befragten nicht beobachten. Vielmehr nimmt der „Priming“ - Mechanismus an, dass kognitive Inhalte (z. B. Erinnerungen an angenommene Freundesmeinungen über Juden) bei der Beantwortung vorangegangener Fragen automatisch aktiviert werden, und dass diese aufgrund ihrer leichten Abrufbarkeit als bedeutsam eingestuften Informationen dann den Antwortprozess der Folgefragen - auch ohne die bewusste Wahrnehmung des Befragten - beeinflussen (Strack 1992, S. 24 f.). Bei den Befragten in der Kontrollgruppe beruhen dagegen die Antworten auf einem vielfältigen Set von Informationen und Wissensgehalten, die Meinung der Freunde wird jedoch nicht explizit bei der Antwortbildung aktiviert.

17 In den vorherigen Abschnitten wurde argumentiert, dass bei der Beantwortung von heiklen Fragen zu Antisemitismus sozial erwünschtes Antwortverhalten zu erwarten ist, d.h. (zumindest) eine Teilmenge der antisemitisch eingestellten Befragten wird demnach im Rahmen einer Befragung die Befragungssituation als öffentliche Situation deuten, ihre Antworten in Richtung der öffentlichen Norm des Anti-Antisemitismus verzerren und damit systematisch ein geringeres Ausmaß an antisemitischen Einstellungen kommunizieren als tatsächlich vorhanden ist. Wird nun die kognitive Verfügbarkeit der privaten Primärgruppennormen (durch einen gezielten Einsatz von „Kontexteffekten“) verstärkt, dann ist mit einer Verringerung der Differenz zwischen tatsächlichen und kommunizierten Einstellungen bei antisemitisch eingestellten Befragten zu rechnen. In diesem Sinne sind eine Zunahme von sozial unerwünschten Antworten und damit ein Rückgang des „Underreporting“ von antisemitischen Einstellungen zu erwarten. 
antisemitischen Einstellungen beantwortet und die Kontrollgruppe die Fragen in umgekehrter Abfolge vorgelegt bekommt:

H1: Der Zusammenhang zwischen der Primärgruppennorm und den kommunizierten, eigenen antisemitischen Einstellungen wird sich verstärken, wenn die Fragen zur Primärgruppennorm vor den Fragen zu den eigenen antisemitischen Einstellungen gestellt werden.

H2: Personen mit einer antisemitischen Primärgruppe werden im Durchschnitt mehr eigene antisemitische Einstellungen kommunizieren, wenn die Fragen zur Primärgruppennorm vor den Fragen zu den eigenen antisemitischen Einstellungen gestellt werden.

\subsection{Weitere Einflussfaktoren des kommunizierten Antisemitismus}

Es wurde postuliert, dass die Bereitschaft antisemitische Einstellungen zu kommunizieren, mit der kognitiven Verfügbarkeit entsprechender Primärgruppennormen variiert. Darüber hinaus können nun weitere Determinanten des kommunizierten Antisemitismus betrachtet werden. So kann der robuste und starke Zusammenhang zwischen Bildung und antisemitischen Einstellungen als ein Desiderat der empirischen Forschung gelten (vgl. Sallen 1977; Bergmann und Erb 1991b; Wittenberg und Schmidt 2004). Damit eng verknüpft kann auch die Wirkung von politischem Interesse auf die Einstellungen der Befragten theoretisch begründet werden, denn neben Bildungsinstitutionen wird die öffentliche Norm des Anti-Antisemitismus auch über den medialen Diskurs an die Bevölkerung vermittelt. Als ein weiterer gesicherter Befund der Antisemitismusforschung kann zudem der starke Zusammenhang von rechtsgerichteten politischen Einstellungen und antisemitischen Einstellungen gelten (vgl. Bergmann 2001; Heitmeyer 2005; Erb 2007). Wir halten deshalb mit Bildung, politischem Interesse und politischer Einstellung drei Variablen fest, die neben ihrem Einfluss auf die tatsächlichen Einstellungen der Befragten, insbesondere auch auf die Kommunikation von antisemitischen Einstellungen wirken. ${ }^{18}$ Folgende Hypothesen lassen sich hierzu formulieren:

H3: Je höher die Bildung einer Person ist, desto weniger antisemitische Einstellungen wird sie kommunizieren.

H4: Je größer das politische Interesse einer Person ist, desto weniger antisemitische Einstellungen wird sie kommunizieren.

H5: Je rechtsgerichteter die allgemeinen politischen Einstellungen einer Person sind, desto mehr antisemitische Einstellungen wird sie kommunizieren.

Im Zusammenhang mit der Wirkung von Bildung weist Heyder (2003) darauf hin, dass der direkte Effekt auf gruppenbezogene Menschenfeindlichkeit (inkl. Antisemitismus) hinterfragt werden muss. Möglicherweise, so Heyder, sind verschiedene indirekte Mechanismen verantwortlich für die klaren Zusammenhänge, die in quantitativen Untersuchungen festgestellt wurden. Die Ergebnisse eines Pfadmodells bestätigen schließlich,

18 Diese Differenzierung ist wichtig und notwendig, da in den empirischen Studien streng genommen nur der kommunizierte Antisemitismus abgebildet wird. 
dass kognitive Fähigkeiten, sozialer Status und Wertorientierungen als Mediatoren wirken können. Aus dem Blickwinkel des Kommunikationslatenz-Ansatzes ergibt sich ein weiterer möglicher indirekter Effekt. Es kann vermutet werden, dass Bildung und auch das Interesse am politischen Geschehen möglicherweise zu einem Teil nicht auf die tatsächlichen antisemitischen Einstellungen der Befragten direkt, sondern vielmehr auf die Kommunikation dieser Einstellungen wirken. Dies könnte nun aber zu der Vermutung führen, dass der in der bisherigen Forschung konstatierte Zusammenhang zwischen diesen Determinanten und antisemitischen Einstellungen überschätzt wurde.

Nehmen wir hierzu in einem Gedankenexperiment an, dass das tatsächliche Ausmaß an antisemitischen Einstellungen bei hoch gebildeten Befragten nur geringfügig niedriger wäre als bei Befragten mit niedriger Bildung. Nehmen wir zudem an, dass die öffentliche Norm gegen antisemitische Äußerungen bei Befragten mit hohem Bildungsniveau stärker wirke, als bei niedrig Gebildeten, weil erstere sich der öffentlichen Norm des AntiAntisemitismus klarer bewusst sind. Gemäß diesen Annahmen werden höher gebildete Befragte mit einer höheren Wahrscheinlichkeit als niedriger gebildete Befragte sozial erwünscht antworten, indem sie in öffentlichen Situationen (wie z. B. einer Befragung) keine antisemitischen Einstellungen kommunizieren, auch wenn diese tatsächlich vorliegen sollten. Der relativ höhere Anteil an sozial erwünschten Antworten in der Gruppe der hoch gebildeten Befragten würde somit zu einer Überschätzung der Einflussstärke der Variable Bildung auf das unbeobachtete Einstellungskonstrukt „Antisemitismus“ führen. Dasselbe gilt für das Interesse am politischen Geschehen.

Möglicherweise lässt sich über die Annahme von sozial erwünschtem Antwortverhalten in Befragungen (und öffentlichen Situationen im Allgemeinen) sogar ein noch grundlegenderer Befund der bisherigen Antisemitismusforschung relativieren, nämlich der starke Zusammenhang von rechtsgerichteten politischen Einstellungen und antisemitischen Einstellungen. Im Anschluss an das obige Gedankenexperiment könnte postuliert werden, dass die öffentliche Norm gegen antisemitische Äußerungen bei „Linken“ größere Wirkung zeige, als bei „Rechten“, die von vornherein ihre Einstellung zum Antisemitismus (und auch entsprechende öffentliche Äußerungen) als positiv besetzten ,,Tabubruch“ verstehen. Folglich werden „Linke“ mit einer höheren Wahrscheinlichkeit als „Rechte“ sozial erwünscht antworten, indem sie in öffentlichen Situationen keine antisemitischen Einstellungen kommunizieren, auch wenn diese tatsächlich vorliegen. In privaten Situationen könnten „Linke“ jedoch ebenso tendenziell antisemitische Aussagen tolerieren oder selbst äußern. ${ }^{19}$ Vor diesem Hintergrund wurde in bisherigen Untersuchungen somit

19 Das Äußern antisemitischer (und anderer ,,politisch inkorrekter") Einstellungen im öffentlichen Raum ist in gewissem Maße geradezu Grundlage der radikalen Selbstbestimmung des Rechtsextremismus. Die Primärgruppe scheint hier geradezu ein öffentliches Bekenntnis zum Antisemitismus zu fordern: „Aufgrund ihres Nazismus und Nationalismus haben diese [rechtsradikalen] Gruppen gar keinen Bedarf und kein Verständnis für die Schutzfunktion der Kommunikationslatenz“ (Bergmann und Erb 1986, S. 231; vgl. auch Heitmeyer 2005). „Linke“ hingegen scheuen sich eventuell, vorhandene antisemitische Einstellungen öffentlich zu äußern, weshalb sie darauf angewiesen sind, sie z. B. über Ersatzkommunikationen zu verbalisieren (Bergmann und Erb 1986, S. 230 ff.). Zum Antisemitismus in der Linken vgl. auch Brumlik et al. (1998), Haury (2002) und Brosch et al. (2006). 
möglicherweise auch die Stärke des Zusammenhangs von allgemeinen politischen Einstellungen und antisemitischen Einstellungen überschätzt.

Mittels einer experimentellen Studie zeigen wir, dass die kognitive Aktivierung einer antisemitischen Primärgruppennorm sozial erwünschtes Antwortverhalten verringert und in einem höheren Maße zu selbstberichtetem Antisemitismus führt. Zudem nehmen wir an, dass sich durch den Einfluss der privaten Primärgruppennormen das Ausmaß sozial erwünschten Antwortverhaltens bei denjenigen Befragten stärker verringern wird, bei denen a priori ein höheres Potenzial für sozial erwünschtes Antwortverhalten erwartet werden kann (höher gebildete, politisch interessierte oder links eingestellte Befragte). Die statistische Kontrolle der Primärgruppennorm und die kognitive Verfügbarkeit derselben sollten sich demnach auch auf die in den Hypothesen 3-5 postulierten Zusammenhänge zwischen dem kommunizierten Antisemitismus und den (jeweiligen) unabhängigen Variablen auswirken.

\section{Empirische Studie}

\subsection{Forschungsdesign}

Um nun die vorgeschlagenen Determinanten des kommunizierten Antisemitismus und die postulierten Effekte der erhöhten kognitiven Verfügbarkeit der Primärgruppennorm auf das Antwortverhalten empirisch zu testen, wird ein randomisiertes Experiment durchgeführt. Hierbei wird eine Hälfte der Befragten zunächst zu den wahrgenommenen antisemitischen Einstellungen „eines Freundes/einer Freundin“ und anschließend zu den eigenen antisemitischen Einstellungen befragt. Der anderen Hälfte der Befragten werden die Fragebatterien in umgekehrter Reihenfolge vorgelegt. ${ }^{20}$

Durch das randomisierte Experimentaldesign und die Zufallszuweisung zu den beiden Gruppen werden a) das unbeobachtete, tatsächliche Ausmaß antisemitischer Einstellungen (sowie alle weiteren Drittvariablen wie Bildung und politisches Interesse) zwischen den beiden Gruppen konstant gehalten, und b) die kognitive Verfügbarkeit der Primärgruppennorm experimentell manipuliert. Das Prinzip der Randomisierung stellt sicher, dass es keine systematischen Unterschiede zwischen den beiden Experimentalgruppen gibt und kontrolliert zudem neben bekannten Drittvariablen auch den Einfluss von

20 Die genaue Operationalisierung lautet wie folgt: „Die nächsten Fragen beschäftigen sich nun mit einem völlig anderen Thema, nämlich mit der Stellung von Juden in der heutigen Welt. Zunächst geht es aber erst einmal nicht um Deine eigene Meinung, sondern um die einer Freundin oder eines Freundes. Falls Du nicht genau weißt, was er oder sie antworten würde, stell Dir folgende Situation vor: Ihr sitzt zusammen vorm Fernseher und ein bekannter Schauspieler sagt in einem Interview einen der folgenden Sätze. Was denkst Du, inwieweit würde Dein Freund oder Deine Freundin diesen Aussagen zustimmen?“ (Experimentalkondition) und „Nun folgen noch einmal die gleichen Aussagen, jetzt geht es aber um die Meinung Deiner Freundin oder Deines Freundes. Falls Du nicht genau weißt, was er oder sie antworten würde, stell Dir folgende Situation vor: Ihr sitzt zusammen vorm Fernseher und ein bekannter Schauspieler sagt in einem Interview einen der folgenden Sätze. Was denkst Du, inwieweit würde Dein Freund oder Deine Freundin diesen Aussagen zustimmen?“ (Kontrollkondition). 
unbekannten Einflussfaktoren, die in den Modellen nicht enthalten sind. Die abhängige Variable ist die kommunizierte antisemitische Einstellung der Befragten. Unterschiede im berichteten Antisemitismus zwischen den Gruppen können somit kausal auf den Faktor kognitive Verfügbarkeit von Primärgruppennormen zurückgeführt werden. Die Primärgruppennorm wurde in Form der selbst wahrgenommenen Meinung einer Freundin oder eines Freundes zu antisemitischen Aussagen erhoben, da im Alter der Befragten Freunde als primäre Bezugsgruppe noch vor der Familie angenommen werden. Unserer Operationalisierung der Primärgruppennorm über ,die Meinung eines Freundes oder einer Freundin“ liegt die Annahme zugrunde, dass bei den geforderten Bewertungen in erster Linie Erinnerungen an ,typische“ oder ,durchschnittliche“ Freunde, die für die Meinungen im eigenen Freundeskreis repräsentativ sind, aktiviert werden. In diesem Zusammenhang erwarten wir eine Angleichung der eigenen Antwort an die zuvor aktivierten Freundesmeinungen. ${ }^{21}$

Die folgende Studie wurde im April 2008 an Mittelschulen und Gymnasien in Chemnitz, Dresden und Leipzig durchgeführt. 241 Schüler der 9. und 10. Klasse wurden mittels eines schriftlich auszufüllenden Fragebogens unter Anwesenheit der gleichzeitig befragten Mitschüler, der Fachlehrer und des Experimentalleiters im Klassenzimmer befragt. ${ }^{22}$

\subsection{Deskriptive Verteilung der Variablen}

Betrachten wir zunächst die deskriptive Verteilung der soziodemografischen Variablen. Das Alter wurde mit der offenen Frage „Wie alt bist du?“ abgefragt. Die jüngsten Befragten gaben an vierzehn (3\%), die ältesten achtzehn Jahre (2\%) alt zu sein. $46 \%$ der Schüler gaben ein Alter von fünfzehn, $33 \%$ ein Alter von sechzehn und 16\% ein Alter von siebzehn Jahren an. Hinsichtlich der Variable Gender (das angegebene soziale Geschlecht) setzt sich die Stichprobe zu 52\% aus Jungen und zu 48\% aus Mädchen zusammen. Bildung wurde über die Frage ,welchen Schulabschluss wirst Du voraussichtlich machen?““

21 Um eine genaue Vorhersage über die Richtung des Effekts formulieren zu können, müssen zusätzliche Annahmen getroffen werden. Befragungspsychologische Studien zeigen, dass es für die Vorhersage der Effektrichtung bedeutsam ist, welchen Vergleichsstandard die Befragten bei der Beantwortung der Folgefrage (oder bei der Selbsteinschätzung) zugrunde legen (vgl. Tourangeau et al. 2000). Wenn der ,typische“ Wert der zu beurteilenden Kategorie als kognitiver Anker aktiviert wird - in diesem Fall würde der Befragte die Meinung eines Freundes einschätzen, den er als repräsentativ für seinen Freundeskreis annimmt - dann ist mit einer Angleichung der Antwort der Zielfrage an die Antwort der vorherigen Frage zu rechnen. Wenn dagegen ein „extremer" Wert der zu beurteilenden Dimension als Vergleichsstandard aktiviert wird - hier würde der Befragte an einen extremen Freund denken, dessen Meinung zu Juden er nicht unbedingt als typisch für seinem übrigen Freundeskreis empfindet - dann ist mit einer Kontrastierung der eigenen, in der nachfolgenden Frage erhobenen Meinung, an diesen Vergleichsstandard zu rechnen (Tourangeau et al. 2000, S. 213).

22 Als Auswahlgrundlage diente eine Liste mit allen Mittelschulen (Hauptschulabschluss oder Realschulabschluss) und Gymnasien in Dresden, Chemnitz und Leipzig. Die Gymnasien und Mittelschulen wurden in alphabetischer Reihenfolge angerufen. Die zu befragenden Klassen wurden durch die Schulleitung ausgewählt. An der Befragung nahmen alle Schüler teil, die eine Einverständniserklärung der Eltern vorlegen konnten. Dies waren ca. 95\% aller Mitglieder der ausgewählten Klassenverbände. 
Tab. 1: Empirische Häufigkeitsverteilung des eigenen Antisemitismus

\begin{tabular}{|c|c|c|c|c|c|c|}
\hline & $\begin{array}{l}\text { stimme } \\
\text { überhaupt } \\
\text { nicht zu }\end{array}$ & $\begin{array}{l}\text { stimme } \\
\text { nicht zu }\end{array}$ & $\begin{array}{l}\text { stimme } \\
\text { eher nicht } \\
\mathrm{zu}\end{array}$ & $\begin{array}{l}\text { stimme } \\
\text { eher zu }\end{array}$ & $\begin{array}{l}\text { stimme } \\
\mathrm{zu}\end{array}$ & $\begin{array}{l}\text { stimme } \\
\text { sehr zu }\end{array}$ \\
\hline $\begin{array}{l}\text { I. Die Juden arbeiten mehr als } \\
\text { andere Menschen mit üblen Tricks, } \\
\text { um das zu erreichen, was sie wollen } \\
\text { (Decker und Brähler 2006) }\end{array}$ & 39 & 23 & 17 & 11 & 5 & 5 \\
\hline $\begin{array}{l}\text { II. Juden haben in der Welt zu viel } \\
\text { Einfluss (Bergmann und Erb 1991b) }\end{array}$ & 25 & 24 & 31 & 9 & 6 & 5 \\
\hline $\begin{array}{l}\text { III. Ich finde es gut, dass wieder } \\
\text { mehr Juden in Deutschland leben }\end{array}$ & 10 & 7 & 15 & 31 & 22 & 15 \\
\hline $\begin{array}{l}\text { IV. Die jüdische Kultur muss gegen } \\
\text { ihre Feinde geschützt werden }\end{array}$ & 7 & 8 & 16 & 26 & 27 & 16 \\
\hline $\begin{array}{l}\text { V. Durch ihr Verhalten sind die } \\
\text { Juden an ihren Verfolgungen } \\
\text { mitschuldig (Heyder et al. 2005) }\end{array}$ & 50 & 18 & 11 & 12 & 5 & 4 \\
\hline
\end{tabular}

Erläuterung: Relative Häufigkeiten in Prozent. Aufgrund vereinzelter fehlender Angaben schwanken die Fallzahlen bei den einzelnen Aussagen zwischen 237 und 241. Codierung: $1=$,stimme überhaupt nicht zu“; $6=$,stimme sehr zu“"

erhoben. $18 \%$ der Befragten antworteten, sie rechnen mit einem Hauptschulabschluss, $30 \%$ mit einem Realschulabschluss, 3\% mit dem Bestehen der Fachhochschulreife und $48 \%$ streben die allgemeine Hochschulreife an.

Antisemitische Einstellungen wurden über Items gemessen, die vorwiegend aus der aktuellen empirischen Antisemitismus- und Rechtsextremismusforschung übernommen wurden. Die positiv formulierten Aussagen III und IV (s. Tab. 1) wurden von uns erarbeitet und $\mathrm{zu}$ den sonst negativ formulierten Items hinzugefügt, um möglichen Zustimmungstendenzen seitens der Befragten entgegenzuwirken (vgl. Schnell et al. 2005). Während die Items III und IV die Teildimension der sozialen Distanz berühren, beziehen sich die übrigen drei Items in erster Linie auf Stereotypen. ${ }^{23}$ Die empirische Verteilung der einzelnen Items ist in Tab. 1 dargestellt.

Die Verteilungen der einzelnen Items sind recht homogen. Bei den negativ formulierten Fragen stimmen in etwa $20 \%$ der befragten Schüler den antisemitischen Aussagen zu, bei den positiv formulierten Items antworten in etwa $30 \%$ der Befragten ablehnend.

Um die Validität und Reliabilität des Gesamtkonstruktes zu überprüfen, wurde eine Skala aus den fünf Items gebildet. Hierbei ergeben jeweils die Summe der Einschätzungen der Items und die anschließende Division dieser Summe durch die Anzahl der Items den Skalenwert jedes Befragten. Die Faktorenanalyse nach der Umpolung der Items III und IV zeigt einen einzigen Faktor mit einem Eigenwert über 1 (genau 3,23) an, der eine erklärte Varianz von 64,68\% aufweist. Die einzelnen Faktorladungen finden sich

23 Bergmann und Erb (1991b, S. 43) unterscheiden beim Antisemitismus die klassischen Einstellungsdimensionen (vgl. Rosenberg und Hovland 1960): Stereotype oder kognitive Überzeugungen, affektive Bewertungen und Handlungsabsichten (bestehend aus sozialer Distanz und passiver Diskriminierung). 
Tab. 2: Faktorenanalyse zu Antisemitismus

\begin{tabular}{lll}
\hline & $\begin{array}{l}\text { Faktorladungen } \\
\text { eigener Antisemitismus }\end{array}$ & $\begin{array}{l}\text { Faktorladungen } \\
\text { Antisemitismus der } \\
\text { Freunde }\end{array}$ \\
\hline $\begin{array}{l}\text { Die Juden arbeiten mehr als andere Menschen mit } \\
\text { üblen Tricks, um das zu erreichen, was sie wollen. } \\
\text { (Item I) }\end{array}$ & 0,806 & 0,796 \\
$\begin{array}{l}\text { Juden haben in der Welt zu viel Einfluss. (Item II) } \\
\begin{array}{l}\text { Ich finde es gut, dass wieder mehr Juden in } \\
\text { Deutschland leben. (Item III) }\end{array}\end{array}$ & 0,739 & 0,734 \\
$\begin{array}{l}\text { Die jüdische Kultur muss gegen ihre Feinde ge- } \\
\text { schützt werden. (Item IV) }\end{array}$ & 0,775 & 0,830 \\
$\begin{array}{l}\text { Durch ihr Verhalten sind die Juden an ihren Ver- } \\
\text { folgungen mitschuldig. (Item V) }\end{array}$ & 0,849 & 0,710 \\
\hline
\end{tabular}

in Tab. 2. Die Reliabilitätsanalyse ergibt ein Cronbachs Alpha von 0,86. Die deskriptive Analyse der Kurzskala ergibt einen Mittelwert von 2,6, einen Median von 2,4 und eine Standardabweichung von 1,2 Skalenpunkten. Die Skala hat einen Wertebereich von 1 (kein Antisemitismus) bis 6 (hoher Antisemitismus).

Die Skala zur antisemitischen Einstellung der Freunde wurde analog zu jener der Eigenmeinung gebildet, ergibt wiederum einen Faktor mit einem Eigenwert über 1 (genau 3,09) mit einer erklärten Varianz von $61,76 \%$ und besteht den Reliabilitätstest mit einem Cronbachs Alpha von 0,85. Die Skala hat einen Mittelwert von 2,9, der Median liegt bei 2,6 und die Standardabweichung bei 1,1 Skalenpunkten. Auf der Basis der konstruierten Skala lassen sich Aussagen über die Verteilung antisemitischer Einstellungen in der Stichprobe treffen. Demnach können 70\% der befragten Schüler (und $61 \%$ der Freunde) als vorurteilsfrei bezeichnet werden (Werte kleiner gleich 3), 19\% der Befragten (24\% der Freunde) haben schwach antisemitische Einstellungen (Werte größer als 3 und kleiner gleich 4 ) und $11 \%$ (15\% der Freunde) haben stark antisemitische Einstellungen (Werte größer 4). ${ }^{24}$

Neben der Bildung werden mit ,politischem Interesse“ und ,politischer Einstellung“ zwei weitere zentrale Determinanten eingeführt. „Politisches Interesse“ wurde mit den beiden Fragen „Mit deiner Familie, Freunden und Bekannten: Wie häufig diskutierst Du da über Politik?“ und „Wie häufig schaust Du Nachrichtensendungen (Fernsehen oder Internet) oder liest die Nachrichten in Zeitungen?" erfasst. Die empirischen Verteilungen der Kategorien sind in Tab. 3 abgebildet. Die Ausprägungen wurden mit 1 (,nie“) bis 5 (,sehr häufig“) codiert.

Als Mittelwert und Median ergeben sich für die obere Frage Werte von 2,8 bzw. 3,0 und für die untere Frage Werte von 3,7 bzw. 4,0. Die Standardabweichungen liegen bei 1,1 bzw. 1,0. Die Rezeption des politischen Diskurses ist damit deutlich ausgeprägter als die Partizipation an politischen Diskussionen.

24 Diese Werte unserer Stichprobe bewegen sich im Rahmen von repräsentativen Umfragen für das Bundesgebiet (vgl. Wittenberg und Schmidt 2004). 
Tab. 3: Empirische Häufigkeitsverteilung des politischen Interesses

\begin{tabular}{|c|c|c|c|c|c|}
\hline & nie & selten & mittel & häufig & sehr häufig \\
\hline $\begin{array}{l}\text { Mit deiner Familie, Freunden und } \\
\text { Bekannten: Wie häufig diskutierst Du } \\
\text { da über Politik? }\end{array}$ & 11 & 30 & 30 & 21 & 8 \\
\hline $\begin{array}{l}\text { Wie häufig schaust Du Nachrichtensendungen } \\
\text { (Fernsehen oder Internet) oder liest die } \\
\text { Nachrichten in Zeitungen? }\end{array}$ & 1 & 11 & 27 & 40 & 21 \\
\hline
\end{tabular}

Erläuterung: Relative Häufigkeiten in Prozent. Die Fallzahlen liegen beim ersten Item bei 241 und beim zweiten bei 237. Codierung: $1=$,nie“ $5=$,sehr häufig“

Die Selbsteinstufung der politischen Position des Befragten erfolgte über eine 10-stufige Antwortskala ${ }^{25}$, deren Mittelwert und Median bei 4,9 bzw. 5,0 liegt. Die Standardabweichung beträgt 1,9 .

\subsection{Test der Hypothesen}

Im Folgenden werden wir die aufgestellten Hypothesen anhand der von uns erhobenen Stichprobe empirisch überprüfen. Dabei sollen zunächst bivariate Pearsonkorrelationen und Mittelwertvergleiche gerechnet und im Anschluss mit Hilfe von multiplen OLSRegressionen die Erklärungskraft unseres Modells getestet werden.

Hypothese 1 nimmt an, dass sich der Zusammenhang zwischen berichteter Eigenmeinung und der Primärgruppennorm verstärkt, wenn vorher die Primärgruppennorm aktiviert wurde. Der Korrelationswert (Pearsons r) zwischen den Skalen zum eigenen Antisemitismus und den Einstellungen der Freunde für die Variante, in der zuerst die eigenen Einstellungen abgefragt wurden, beträgt $r=0,75(p=0,00)$ gegenüber einem Wert von $r=0,86(p=0,00)$ für die Fragebogenvariante, in der zuerst nach den Einstellungen der Freunde gefragt wurde. ${ }^{26}$ Die eigene Meinung passt sich also wie angenommen stärker an die Primärgruppennorm an, wenn letztere vorher kognitiv aktiviert wurde. ${ }^{27}$

Hypothese 2 postuliert, dass Befragte mit einer antisemitischen Primärgruppe, bei denen die Primärgruppennormen vor der Beantwortung der Fragen nach den eigenen Ein-

25 Wortlaut der Frage: „Viele Menschen verwenden die Begriffe „rechts“ und „links“, wenn es darum geht, unterschiedliche politische Einstellungen zu kennzeichnen. Wenn Du an Deine eigenen politischen Ansichten denkst, wo würdest Du diese Ansichten auf einer Skala von 1 bis 10 einstufen, wobei $1=$ links und $10=$ rechts bedeutet?"

26 Die recht hohen Korrelationswerte zwischen den Skalen zum eigenen Antisemitismus und den Einstellungen der Freunde können als Indiz für die von uns postulierten Anpassungsprozesse gewertet werden, wonach eine Angleichung der eigenen Antwort an die zuvor aktivierte Meinung eines „typischen“ Freundes stattfindet. Würden dagegen, als alternative Hypothese, primär die Meinungen von ,extremen“ Freunden aktiviert werden, dann würde man (anstatt einer Angleichung) eher eine Kontrastierung der eigenen Meinung, an der zuvor aktivierten Primärgruppennorm erwarten. Dies würde insgesamt zu schwächeren Korrelationen führen (vgl. hierzu auch die Ausführungen in Fußnote 21).

27 Bei einer umgekehrten Kausalrichtung, der Projektion der eigenen Einstellung auf die angenommene Norm der Primärgruppe, wäre dagegen in der Kontrollgruppe ein im Vergleich zur Experimentalgruppe stärkerer Zusammenhang zu erwarten gewesen. 
stellungen kognitiv aktiviert wurden, antisemitischen Aussagen eher zustimmen werden, als jene, bei denen die Primärgruppennormen nicht aktiviert wurden. Für den empirischen Test der Hypothese betrachten wir deshalb zunächst nur die Befragten, deren Freunde schwach oder stark antisemitisch eingeschätzt werden (vgl. hierzu die Klassifikation im vorherigen Abschnitt). Bei Ausschluss der vorurteilsfreien Freunde (dies sind $61 \%$ der Befragten) unterscheiden sich die Mittelwerte der Skala zum eigenen Antisemitismus um 0,7 Skalenpunkte $(\mathrm{p}=0,00)$ : Sie betragen 3,3 bei der Kontrollgruppe $(\mathrm{N}=49)$ und 4,0 bei der Experimentalgruppe $(\mathrm{N}=40) .{ }^{28}$ Dagegen lassen sich bei den Befragten mit „Vorurteilsfreien“ Freunden wie erwartet keine signifikanten Unterschiede feststellen: Die Mittelwerte betragen hier 1,9 bei der Kontrollgruppe $(\mathrm{N}=63)$ und 2,0 bei der Experimentalgruppe $(\mathrm{N}=77)$. Hypothese 2 kann damit belegt werden.

Der Zusammenhang von Bildung ${ }^{29}$ und der Bereitschaft antisemitische Einstellungen zu kommunizieren, wie ihn Hypothese 3 postuliert, ist wie erwartetet nachweisbar. Zwischen Bildung und Antisemitismus kann ein negativer Zusammenhang ( $\mathrm{r}=-0,47$; $\mathrm{p}=0,00)$ festgestellt werden. Dieser Befund wurde in mehreren Untersuchungen bereits bestätigt (Sallen 1977; Wittenberg und Schmidt 2004; Krumpal 2010), gilt jedoch nur für die nach 1945 sozialisierten Kohorten (vgl. Bergmann und Erb 1991b).

Eher uneinheitlich fallen dagegen die Werte für Hypothese 4 aus, die politisches Interesse und Antisemitismus in Beziehung setzen. Betrachtet man zunächst die Rezeption des politischen Geschehens durch mediale Berichterstattung (,Wie häufig schaust oder liest Du Nachrichten?"), so lässt sich kein Zusammenhang zur Kommunikation antisemitischer Einstellungen feststellen $(r=-0,02 ; \mathrm{p}=0,80)$. Die Partizipation an politischen Diskussionen - ein stärkerer Indikator für politisches Interesse - zeigt einen nur geringen Zusammenhang $(r=-0,16 ; p=0,01)$, der in die angenommene Richtung weist. Hinsichtlich des Interesses am politischen Geschehen unterscheiden sich Personen, die antisemitische Einstellungen kommunizieren, nur geringfügig von Personen, die keine antisemitischen Einstellungen kommunizieren.

Der in Hypothese 5 angenommene Zusammenhang kann hingegen bestätigt werden. Die Links-Rechts-Selbsteinstufung und die Skala zum eigenen Antisemitismus korrelieren recht stark $(r=0,60 ; p=0,00)$. Dieser Befund stützt die weitläufig geteilte Annahme, dass ideologisch komplexer Antisemitismus heute in Deutschland vorwiegend von rechtsextrem eingestellten Personen offen kommuniziert wird (vgl. z. B. Heitmeyer 2005).

Auf welcher Ebene (Kommunikations- oder Einstellungsebene) nun jedoch die beobachteten Zusammenhänge zwischen den Antwortmustern der Befragten verankert sind, bleibt an dieser Stelle noch unklar. Einen Hinweis darauf kann erst die multivariate Auswertung des experimentellen Versuchaufbaus bringen. In einem weiteren Schritt werden wir aus diesem Grund und um die Erklärungskraft unseres Modells zu bestimmen, unsere Hypothesen im Rahmen von multiplen linearen Regressionsmodellen empirisch überprü-

28 Höhere Werte zeigen eine höhere Ausprägung antisemitischer Einstellungen an. Die Experimentalgruppe ist jene Gruppe, bei der die Freundesmeinung kognitiv aktiviert wurde.

29 Die Bildungsvariable (angestrebter Bildungsabschluss) wurde umcodiert, sodass „kein Abschluss“ einem Wert von 0 entspricht, „Hauptschulabschluss“ einem Wert von 1, „Realschulabschluss“ einem Wert von 2, „Fachhochschulreife“ einem Wert von 3 und ,allgemeines Abitur" einem Wert von 4. 
Tab. 4: Determinanten des selbstberichteten Antisemitismus (multiple OLS-Regressionen)

\begin{tabular}{llll}
\hline & Modell 1 & Modell 2 & Modell 3 \\
\hline $\begin{array}{l}\text { Bildung } \\
(0=\text { kein Abschluss; 4=Abitur) }\end{array}$ & $-0,28^{*}$ & $-0,29^{*}$ & $-0,13^{*}$ \\
$\begin{array}{l}\text { Diskussionen über Politik } \\
\text { (Politisches Interesse) }\end{array}$ & $(-5,58)$ & $(-5,69)$ & $(-3,27)$ \\
$(1=$ nie; 5=sehr häufig) & $-0,14^{*}$ & $-0,14^{*}$ & $-0,03$ \\
$\begin{array}{l}\text { Nachrichtenkonsum } \\
\text { (Politisches Interesse) }\end{array}$ & $(-2,51)$ & $(-2,48)$ & $(-0,58)$ \\
$(1=$ nie; 5=sehr häufig) & & & \\
$\begin{array}{l}\text { Politische Einstellung } \\
(1=\text { links; 10=rechts) }\end{array}$ & 0,03 & 0,03 & 0,01 \\
$\begin{array}{l}\text { Gender } \\
(0=\text { männlich; 1=weiblich) }\end{array}$ & $(0,47)$ & $(0,50)$ & $(0,06)$ \\
$\begin{array}{l}\text { Fragereihenfolge } \\
(0=\text { Kontrollkondition; } \\
1=\text { Experimentalkondition) }\end{array}$ & $0,30^{*}$ & & \\
Primärgruppennorm & $(8,87)$ & $(8,87)$ & $(4,79)$ \\
$(1=$ kein Antisemitismus; 6=hoher & $-0,18$ & $-0,17$ & $-0,01$ \\
Antisemitismus) & $(-1,49)$ & $(-1,41)$ & $(-0,03)$ \\
Fragereihenfolge $\times$ Primärgruppennorm & & $0,20^{+}$ & $-0,58^{*}$ \\
& & $(1,69)$ & $(-2,42)$ \\
Konstante & & & \\
& & & $0,49^{*}$ \\
Adjusted R & & & $(8,00)$ \\
N & & & $0,31^{*}$ \\
\hline
\end{tabular}

Erläuterung: ${ }^{*}$ signifikantaufdem $5 \%$-Niveau; ${ }^{+}$signifikantaufdem $10 \%$-Niveau. Unstandardisierte Koeffizienten. t-Werte in Klammern; abhängige Variable ist der selbstberichtete Antisemitismus (1=,kein Antisemitismus“; $6=$,,hoher Antisemitismus“)

fen. Die abhängige Variable ist jeweils die Skala zum selbstberichteten Antisemitismus. Das einfachste Modell testet zunächst die Hypothesen 3-5 gemeinsam (s. Modell 1 in Tab.4). Es kann $46 \%$ der Varianz erklären (Adjusted $\mathrm{R}^{2}=0,46$ ). Die Variable politisches Interesse zeigt keinen (Nachrichtenkonsum; Regressionskoeffizient $=0,03 ; \mathrm{p}=0,64$ ) oder nur einen schwachen (Diskussionen über Politik; Regressionskoeffizient $=-0,14$; $\mathrm{p}=0,01)$ signifikanten Einfluss auf den kommunizierten Antisemitismus. Im Vergleich dazu deuten die statistischen Befunde für die Variablen Bildung (Regressionskoeffizient $=-0,28 ; p=0,00)$ und politische Einstellung (Regressionskoeffizient $=0,30 ; p=0,00)$ wie erwartet, jeweils auf einen starken und signifikanten Zusammenhang mit dem selbstberichteten Antisemitismus hin. Schüler, die höhere Schulabschlüsse anstreben und eher linksgerichtete politische Einstellungen vertreten, kommunizieren weniger antisemitische Einstellungen. Die Ergebnisse der bivariaten Analysen lassen sich somit im Rahmen einer multiplen Regressionsanalyse reproduzieren. 
In den Modellen 2 und 3 wird nun der experimentelle Versuchsaufbau berücksichtigt. Interpretieren wir zunächst die Daten hinsichtlich unserer Hypothesen 1 und 2. Hierzu werden die Variablen Fragereihenfolge, Antisemitismus der Freunde und der entsprechende Interaktionsterm der beiden Variablen in die Modelle eingeführt. Modell 2 (Adjusted $\mathrm{R}^{2}=0,46$ ) zeigt zunächst wie angenommen einen signifikanten Einfluss der Fragereihenfolge (oder der kognitiven Verfügbarkeit der Primärgruppenorm) auf den kommunizierten Antisemitismus (Regressionskoeffizient=0,20; $p=0,09$ ). Die Werte der bereits in Modell 1 enthaltenen Variablen verändern sich nur minimal. Modell 3 (Adjusted $\mathrm{R}^{2}=0,70$ ) bestätigt dann die angenommenen Zusammenhänge aus Hypothese 1 und Hypothese 2 im Detail. Zunächst lässt sich der allgemeine Zusammenhang zwischen Primärgruppennorm und eigener kommunizierter Einstellung nachweisen (Regressionskoeffizient $=0,49 ; \mathrm{p}=0,00)$, der sich nochmals vergrößert, wenn die Primärgruppennorm vorher aktiviert wurde (Regressionskoeffizient des Interaktionseffektes $=0,31 ; p=0,00$ ). Dies wurde in Hypothese 1 postuliert. Der negative Wert des Regressionskoeffizienten der Fragereihenfolge $(-0,58 ; \mathrm{p}=0,02)$ und der eindeutige Effekt des Interaktionsterms (s. oben) weisen, wie in Hypothese 2 angenommen, darauf hin, dass die kognitive Aktivierung der Primärgruppennorm den kommunizierten Antisemitismus nur dann verstärkt, wenn die Primärgruppe hinreichend antisemitische Einstellungen aufweist und die Primärgruppennorm somit der öffentlichen Norm des Anti-Antisemitismus widerspricht.

Im Theorieteil hatten wir ein Gedankenexperiment beschrieben, demzufolge die in Hypothese 3 bis Hypothese 5 eingeführten Determinanten (Bildung, politisches Interesse und die allgemeine politische Einstellung) des Antisemitismus möglicherweise zu einem Teil nicht auf die antisemitischen Einstellungen der Befragten direkt wirken, sondern lediglich auf deren Kommunikation. Die in der bisherigen Forschung konstatierten Zusammenhänge dieser Variablen mit antisemitischen Einstellungen wären demnach überschätzt worden. Unsere Daten stützen diese Vermutung. Alle Regressionskoeffizienten der relevanten Variablen verringern sich im dritten Modell. Der Einfluss der Partizipation an politischen Diskussionen ist dann nicht mehr signifikant (Regressionskoeffizient $=-0,03$; $\mathrm{p}=0,56$ ). Die Effektstärken von Bildung (Regressionskoeffizient $=-0,13 ; \mathrm{p}=0,00$ ) und der allgemeinen politischen Einstellung (Regressionskoeffizient $=0,14 ; p=0,00)$ sinken ebenfalls deutlich. Die Kontrolle der Primärgruppennorm und die kognitive Verfügbarkeit derselben wirken sich demnach klar auf die Zusammenhänge zwischen dem kommunizierten Antisemitismus und den berichteten unabhängigen Variablen aus.

\section{Fazit und Diskussion}

Als ein wichtiges Ergebnis der Studie lässt sich herausheben, dass das Ausmaß der Zustimmung zu antisemitischen Items signifikant von der kognitiven Verfügbarkeit von Primärgruppenormen beeinflusst wird. Ein denkbarer Einwand hierbei wäre, dass es sich bei den hier beobachteten starken Zusammenhängen in der Experimentalgruppe um methodische Artefakte mit einer geringen externen Validität handeln könnte. Diesem Einwand kann mit dem Argument begegnet werden, dass die Primärgruppennorm in alltäglichen Situationen weitaus verfügbarer und somit stärker wirksam ist, als im Rahmen einer Befragung. So können innerhalb eines gleichgesinnten Freundeskreises antisemitische 
Einstellungen ohne größere Befürchtungen vor Sanktionen geäußert werden oder würden sogar positiv verstärkt werden. Zudem sollte es in einem antisemitisch eingestellten Freundeskreis recht schwerfallen, eine anti-antisemitische Einstellung zu vertreten. Man würde in diesem Fall mit einer hohen Wahrscheinlichkeit aus der Gruppe ausgegrenzt werden oder würde von vornherein aus Gründen kognitiver Dissonanz (Festinger 1978) einen anderen Freundeskreis wählen. Vor diesem Hintergrund kann die Einstellung der Freunde als ein guter Prädiktor für die eigene Einstellung angesehen werden. Dagegen scheint es wenig plausibel anzunehmen, dass die öffentliche Norm des Anti-Antisemitismus eine ähnlich starke Wirkung auf die eigene Einstellung ausübt, wenn sie nicht als Primärgruppennorm innerhalb des privaten Bereichs selbst Gültigkeit hat. Somit erscheint es sinnvoll, die Primärgruppennorm vor der Messung antisemitischer Einstellungen als kognitiven Orientierungsanker zu setzen und dadurch das Antwortverhalten der Befragten in Richtung einer höheren Konsistenz mit der Primärgruppennorm zu beeinflussen. Im Unterschied zur Angleichung des eigenen Antwortverhaltens an die öffentliche Norm des Anti-Antisemitismus (oder an die vermeintlichen Erwartungen des Experimentalleiters in der Interviewsituation), deren Zusammenhang mit den tatsächlichen Einstellungen des Befragten als nur schwach angenommen wird, ist bei einer Orientierung des Antwortverhaltens an den Primärgruppennormen, die mit den tatsächlichen Einstellungen des Befragten in der Regel stark zusammenhängen, eine validere Messung antisemitischer Einstellungen zu erwarten.

Unser Beitrag deutet zudem darauf hin, dass die bisher als gesichert angenommenen Determinanten antisemitischer Einstellung, Bildung und allgemeine politische Einstellungen, teilweise über das Kommunikationsverhalten moderiert werden. Dasselbe gilt für das von uns kontrollierte politische Interesse. Diese Ergebnisse stützen die Plausibilität der Theorie der Kommunikationslatenz (Bergmann und Erb 1986), nach welcher antisemitische Einstellungen zu einem gewissen Maße nur aus der Öffentlichkeit gedrängt, nicht aber völlig abgebaut werden. Damit gewinnen insgesamt bezugsgruppen- und kommunikationstheoretische Betrachtungen zunehmend an Relevanz für die Antisemitismusforschung.

In zukünftigen Studien zu Antisemitismus sollte der Einfluss sozial erwünschten Antwortverhaltens stärker berücksichtigt werden. Dies kann in Form von kognitiven Stimuli innerhalb des Fragebogens geschehen. Aber auch andere Ansätze, die der konkreten Befragungssituation Relevanz einräumen, sind denkbar. So ließe sich der Einfluss von Primärgruppen auf das Antwortverhalten in einem „natürlichen“ Setting überprüfen. Mit einem Teil der Befragten könnten im Rahmen eines Feldexperimentes Interviews durchgeführt werden, bei denen Mitglieder der Bezugsgruppe des Befragten anwesend sind (vgl. auch die neueren Studien mit Gruppeninterviews von Decker et al. 2008). In der Kontrollgruppe würden dagegen die Befragten ohne Anwesenheit von Bezugsgruppenmitgliedern interviewt werden. Zudem ließe sich neben den Freundesnetzwerken auch der Einfluss anderer Bezugspersonen und -gruppen auf die Kommunikationsbereitschaft von antisemitischen Einstellungen untersuchen (z.B. Familieneinflüsse). Auch hierbei könnten experimentelle Methoden eingesetzt werden.

Danksagung: Die Autoren danken Kurt Mühler, Ulf Liebe sowie den Gutachtern und Herausgebern der KZfSS für anregende Diskussionen bzw. nützliche Überarbeitungshinweise. 


\section{Literatur}

Adorno, Theodor W., Else Frenkel-Brunswik, Daniel J. Levinson, und R. Nevitt Sanford. 1950. The authoritarian personality. New York: Harper and Brothers.

Adorno, Theodor W. 1997. Zur Bekämpfung des Antisemitismus heute. In Theodor W. Adorno. Gesammelte Schriften Band 20/1: Vermischte Schriften I, Hrsg. Rolf Tiedemann und Gretel Adorno, 360-383. Frankfurt a. M.: Suhrkamp.

Adorno, Theodor W., und Max Horkheimer. 1997. Dialektik der Aufklärung. Philosophische Fragmente. In Theodor W. Adorno. Gesammelte Schriften Band 3: Dialektik der Aufklärung. Philosophische Fragmente, Hrsg. Rolf Tiedemann und Gretel Adorno, 7-335. Frankfurt a. M.: Suhrkamp.

Benz, Wolfgang. 2004. Was ist Antisemitismus? Bonn: Bundeszentrale für politische Bildung.

Bergmann, Werner. Hrsg. 1988. Error without trial. Psychological research on antisemitism. Berlin, New York: De Gruyter.

Bergmann, Werner. 2001. Antisemitismus in beiden Teilen Deutschlands von 1945 bis heute. In Rechtsextremismus in der Bundesrepublik Deutschland, Hrsg. Wilfried Schubarth und Richard Stöss, 131-154. Opladen: Leske und Budrich.

Bergmann, Werner, und Rainer Erb. 1986. Kommunikationslatenz, Moral und öffentliche Meinung. Theoretische Überlegungen zum Antisemitismus in der Bundesrepublik Deutschland. Kölner Zeitschrift für Soziologie und Sozialpsychologie 38:223-246.

Bergmann, Werner, und Rainer Erb. 1991a. „Mir ist das Thema Juden irgendwie unangenehm.“ Kommunikationslatenz und die Wahrnehmung des Meinungsklimas im Fall des Antisemitismus. Kölner Zeitschrift für Soziologie und Sozialpsychologie 43:502-519.

Bergmann, Werner, und Rainer Erb. 1991b. Antisemitismus in der Bundesrepublik Deutschland. Ergebnisse der empirischen Forschung von 1946-1989. Opladen: Leske und Budrich.

Bishop, Georg F., Robert W. Oldendick, und Alfred J. Tuchfarber. 1984. What must my interest in politics be if I just told you ,I don't know'? Public Opinion Quarterly 48:510-519.

Broder, Henryk M. 1976. Antizionismus - Antisemitismus von links? Aus Politik und Zeitgeschichte 24:31-46.

Brosch, Matthias, Michael Elm, Norman Geißler, Brigitta Elisa Simbürger, und Oliver von Wrochem. Hrsg. 2006. Exklusive Solidarität. Linker Antisemitismus in Deutschland. Berlin: Metropol.

Brumlik, Micha. 1986. Die Angst vor dem Vater. Judenfeindliche Tendenzen im Umkreis neuer sozialer Bewegungen. In Antisemitismus nach dem Holocaust, Hrsg. Alphons Silbermann und Julius H. Schoeps, 133-162. Köln: Wissenschaft und Politik.

Brumlik, Micha, Doron Kiesel, und Linda Reisch. Hrsg. 1998. Der Antisemitismus und die Linke. Frankfurt a. M.: Haag und Herchen.

Claussen, Detlev. 1994. Grenzen der Aufklärung. Die gesellschaftliche Genese des modernen Antisemitismus. Frankfurt a. M.: Fischer.

Decker, Oliver, und Elmar Brähler. 2006. Vom Rand zur Mitte. Rechtsextreme Einstellung und ihre Einflussfaktoren in Deutschland. Berlin: Friedrich-Ebert-Stiftung.

Decker, Oliver, Katharina Rothe, Marliese Weissmann, Norman Geißler, und Elmar Brähler. 2008. Ein Blick in die Mitte. Zur Entstehung rechtsextremer und demokratischer Einstellungen. Berlin: Friedrich-Ebert-Stiftung.

Diekmann, Andreas. 2008. Empirische Sozialforschung. Grundlagen, Methoden, Anwendungen. 19. Aufl. Reinbek: Rowohlt Taschenbuch.

Diner, Dan. 2004. Der Sarkophag zeigt Risse. Über Israel, Palästina und die Frage eines „,neuen Antisemitismus“. In Neuer Antisemitismus? Eine globale Debatte, Hrsg. Doron Rabinovici, Ulrich Speck, und Natan Sznaider, 310-329. Frankfurt a. M.: Suhrkamp.

Eagly, Alice H., und Shelly Chaiken. 1993. Psychology of attitudes. Fort Worth: Harcourt Brace Jovanovich. 
Erb, Rainer. 2007. Organisierte Antisemiten. Aus Politik und Zeitgeschichte 31:19-26.

Fein, Helen. Hrsg. 1987. The persisting question. Sociological perspectives and social contexts of modern antisemitism. Berlin: De Gruyter.

Feldman, Jack M. 1992. Constructive processes as a source of context effects in survey research: Explorations in self-generated validity. In Context effects in social and psychological research, Hrsg. Norbert Schwarz und Seymour Sudman, 49-61. New York: Springer-Verlag.

Festinger, Leon. 1978. Theorie der kognitiven Dissonanz. Bern: Hans Huber.

Groves, Robert M., Floyd J. Fowler, Jr., Mick P. Couper, James M. Lepkowski, Eleanor Singer, und Roger Tourangeau. 2004. Survey methodology. Hoboken, NJ: Wiley.

Haury, Thomas. 2002. Antisemitismus von links. Kommunistische Ideologie, Nationalismus und Antizionismus in der frühen DDR. Hamburg: Hamburger Edition.

Heider, Fritz. 1977. Psychologie der interpersonellen Beziehungen. Stuttgart: Klett.

Heitmeyer, Wilhelm. 2005. Gruppenbezogene Menschenfeindlichkeit. Die theoretische Konzeption und empirische Ergebnisse aus den Jahren 2002, 2003 und 2004. In Deutsche Zustände. Folge 3, Hrsg. Wilhelm Heitmeyer, 13-36. Frankfurt a. M.: Suhrkamp.

Heyder, Aribert. 2003. Bessere Bildung, bessere Menschen? Genaueres Hinsehen hilft weiter. In Deutsche Zustände. Folge 2, Hrsg. Wilhelm Heitmeyer, 78-99. Frankfurt a. M.: Suhrkamp.

Heyder, Aribert, Julia Iser, und Peter Schmidt. 2005. Israelkritik oder Antisemitismus? In Deutsche Zustände. Folge 3, Hrsg. Wilhelm Heitmeyer, 144-163. Frankfurt a. M.: Suhrkamp.

Homans, George C. 1974. Social behavior: Its elementary forms. New York: Harcourt, Brace, Jovanovich.

Horkheimer, Max. Hrsg. 2005. Schriften des Instituts für Sozialforschung. Band 5: Studien über Autorität und Familie. Lüneburg: Zu Klampen.

Krumpal, Ivar. 2009. Estimating the prevalence of unsocial opinions via the randomized response technique. In Classification and data analysis 2009 - 7th Meeting of the Classification and Data Analysis Group of the Italian Statistical Society, Hrsg. Salvatore Ingrassia und Roberto Rocci, 315-318. Padova: CLEUP.

Krumpal, Ivar. 2010. Sensitive questions and measurement error: Using the randomized response technique to reduce social desirability bias in CATI Surveys. Dissertation, Fakultät für Sozialwissenschaften und Philosophie, Universität Leipzig.

Lee, Raymond M. 1993. Doing research on sensitive topics. London: Sage.

Merton, Robert K. 1957. Social theory and social structure. Glencoe: Free Press.

Nisbett, Richard E., und Timothy DeCamp Wilson. 1977. The halo effect: Evidence for unconscious alteration of judgments. Journal of Personality and Social Psychology 35:250-256.

Opp, Karl-Dieter. 2001. Norms. In International encyclopedia of the social \& behavioral sciences, Hrsg. Neil J. Smelser und Paul B. Baltes, 10714-10720. Amsterdam: Elsevier.

Phillips, Damon J., und Ezra Zuckerman. 2001. Middle-status conformity: Theoretical restatement and empirical demonstration in two markets. American Journal of Sociology 107:379-429.

Postone, Moishe. 1988. Nationalsozialismus und Antisemitismus. Ein theoretischer Versuch. In Zivilisationsbruch. Denken nach Auschwitz, Hrsg. Dan Diner, 242-254. Frankfurt a. M.: Fischer.

Rauhut, Heiko, Ivar Krumpal, und Mandy Beuer. 2009. Rechtfertigungen und Bagatelldelikte: Ein experimenteller Test. In Klein aber fein! Quantitative empirische Sozialforschung mit kleinen Fallzahlen, Hrsg. Peter Kriwy und Christiane Gross, 373-396. Wiesbaden: VS Verlag für Sozialwissenschaften.

Rensmann, Lars. 1998. Kritische Theorie über den Antisemitismus. Studien zu Struktur, Erklärungspotential und Aktualität. Berlin: Argument Verlag.

Rosenberg, Milton J., und Carl I. Hovland. 1960. Cognitive, affective, and behavioral components of attitudes. In Attitude organisation and change, Hrsg. Carl I. Hovland, Milton J. Rosenberg, William J. McGuire, Robert P. Abelson, und Jack W. Brehm, 1-14. New Haven: Yale University Press. 
Sallen, Herbert A. 1977. Zum Antisemitismus in der Bundesrepublik Deutschland. Konzepte, Methoden und Ergebnisse der empirischen Antisemitismusforschung. Frankfurt a. M.: Haag und Herchen.

Salzborn, Samuel. 2010. Antisemitismus als negative Leitidee der Moderne: sozialwissenschaftliche Theorien im Vergleich. Frankfurt a. M.: Campus.

Schaeffer, Nora C., und Stanley Presser. 2003. The science of asking questions. Annual Review of Sociology 29:65-88.

Scherr, Albert, und Barbara Schäuble. 2008. „Wir“ und „die Juden“. Gegenwärtiger Antisemitismus als Differenzkonstruktion. Berliner Debatte Initial 1-2:3-14.

Schnell, Rainer, Paul Hill, und Elke Esser. 2005. Methoden der empirischen Sozialforschung, 7. Aufl. München: Oldenbourg.

Schönbach, Peter. 1961. Reaktionen auf die antisemitische Welle im Winter 1959/60. Frankfurt a. M.: Europäische Verlagsanstalt.

Schwarz, Norbert. 1991. In welcher Reihenfolge fragen. Kontexteffekte in standardisierten Befragungen. ZUMA Arbeitsbericht 16.

Schwarz, Norbert, Hans-Jürgern Hippler, und Elisabeth Noelle-Neumann. 1989. Einflüsse der Reihenfolge von Antwortvorgaben bei geschlossenen Fragen. ZUMA Nachrichten 25:24-38.

Schwarz, Norbert, und Andreas Bayer. 1989. Variation der Fragereihenfolge als Instrument der Kausalitätsprüfung. Eine Untersuchung zur Neutralisationstheorie devianten Verhaltens. ZUMA-Arbeitsbericht 89.

Schwarz, Norbert, und Seymour Sudman. Hrsg. 1992. Context effects in social and psychological research. New York: Springer-Verlag.

Sloman, Steven A., C. A. Gordon Hayman, Nobuo Ohta, Janine Law, und Endel Tulving, 1988. Forgetting in primed fragment completion. Journal of Cognition 14:223-239.

Stocké, Volker. 2004. Entstehungsbedingungen von Antwortverzerrungen durch soziale Erwünschtheit. Ein Vergleich der Prognosen der Rational-Choice-Theorie und des Modells der FrameSelektion. Zeitschrift für Soziologie 33:303-320.

Strack, Fritz, Norbert Schwarz, und Michaela Wänke. 1991. Semantic and pragmatic aspects of context effects in social and psychological research. Social Cognition 9:111-125.

Strack, Fritz. 1992. „Order effects“ in survey research: Activation and information functions of preceding questions. In Context effects in social and psychological research, Hrsg. Norbert Schwarz und Seymour Sudman, 23-34. New York: Springer-Verlag.

Tourangeau, Roger. 1992. Context effects on responses to attitude questions: Attitudes as memory structures. In Context effects in social and psychological research, Hrsg. Norbert Schwarz und Seymour Sudman, 35-47. New York: Springer-Verlag.

Tourangeau, Roger, und Kenneth A. Rasinski. 1988. Cognitive processes underlying context effects in attitude measurement. Psychological Bulletin 103:299-314.

Tourangeau, Roger, Lance J. Rips, und Kenneth A. Rasinski. 2000. The psychology of survey response. Cambridge: Cambridge University Press.

Tourangeau, Roger, und Ting Yan. 2007. Sensitive questions in surveys. Psychological Bulletin 133:859-883.

Van Koolwijk, Jürgen. 1969. Unangenehme Fragen. Paradigma für die Reaktionen des Befragten im Interview. Kölner Zeitschrift für Soziologie und Sozialpsychologie 21:864-875.

Wittenberg, Reinhard, und Manuela Schmidt. 2004. Antisemitische Einstellungen in Deutschland zwischen 1994 und 2002. Eine Sekundäranalyse repräsentativer Bevölkerungsumfragen aus den Jahren 1994, 1996, 1998 und 2002. Jahrbuch für Antisemitismusforschung 13:161-183. 
Heiko Beyer, 1981, M.A., wissenschaftlicher Mitarbeiter am Lehrstuhl für Soziologie Ländlicher Räume an der Georg-August-Universität Göttingen. Forschungsgebiete: Soziologische Theorie, Soziologie der Moderne, Antisemitismus- und Antiamerikanismusforschung. Veröffentlichung: Antiamerikanismus und Antisemitismus: Zum Verhältnis zweier Ressentiments, Zeitschrift für Soziologie 39, 2010 (mit U. Liebe).

Ivar Krumpal, 1975, Dr., wissenschaftlicher Mitarbeiter am Lehrstuhl für Theorie und Theoriegeschichte am Institut für Soziologie der Universität Leipzig. Forschungsgebiete: Soziologische Theorie, Antisemitismusforschung, sozial erwünschtes Antwortverhalten, Methoden der empirischen Sozialforschung, Survey Methodology. Veröffentlichungen: The Framing of Risks and the Communication of Subjective Probabilities for Victimizations, Quality \& Quantity, im Druck (mit H. Rauhut, D. Böhr und E. Naumann); Die Durchsetzung sozialer Normen in Low-Cost und HighCost Situationen, Zeitschrift für Soziologie 37, 2008 (mit H. Rauhut). 